Review Article

\title{
Vitreous Inflammation Associated with Intravitreal Anti-VEGF Pharmacotherapy
}

\author{
Shivi Agrawal, ${ }^{1}$ Malav Joshi, ${ }^{1}$ and John B. Christoforidis ${ }^{2}$ \\ ${ }^{1}$ Department of Ophthalmology, University of Arizona Medical Center, Tucson, AZ 85711, USA \\ ${ }^{2}$ Retina Division, Department of Ophthalmology, University of Arizona Medical Center, 655 N. Alvernon Way, Suite 108, Tucson, \\ AZ 85711, USA \\ Correspondence should be addressed to John B. Christoforidis; jbchristo@hotmail.com
}

Received 5 August 2013; Accepted 25 September 2013

Academic Editor: Mario R. Romano

Copyright (C) 2013 Shivi Agrawal et al. This is an open access article distributed under the Creative Commons Attribution License, which permits unrestricted use, distribution, and reproduction in any medium, provided the original work is properly cited.

\begin{abstract}
Vascular endothelial growth factor (VEGF) is a potent promoter of angiogenesis involved in a wide variety of physiologic processes. Intravitreal injections targeting VEGF have transformed the treatment of neovascular retinal diseases. Currently, there are four antiVEGF agents in use: bevacizumab, ranibizumab, pegaptanib, and aflibercept. The success and frequency of anti-VEGF therapy have made the ocular safety profile of these agents of vital importance. This paper focuses on sterile endophthalmitis. In this paper, we compare the incidences of posttreatment sterile endophthalmitis among the four agents, review the mechanism of actions, and discuss the most prevalent hypotheses leading to sterile endophthalmitis.
\end{abstract}

\section{Introduction}

Vascular endothelial growth factor-A (VEGF) is the master regulator of angiogenesis [1]. Pharmacotherapy utilizing intravitreal injections of antivascular endothelial growth factor (anti-VEGF) agents has revolutionized the treatment of neovascular retinal disorders by inhibiting angiogenesis. Bevacizumab was the first intravitreal agent utilized for the treatment of macular edema secondary to a branch retinal vein occlusion and age-related macular degeneration $[2$, 3]. Today, multiple anti-VEGF agents have been developed including bevacizumab, pegaptanib, ranibizumab, and aflibercept. These agents have shown promising results in the treatment of various retinal diseases including age-related macular degeneration, diabetic retinopathy [4], neovascular glaucoma [5], retinopathy of prematurity [6], and intraocular tumors [7]. Today, the use of intravitreal anti-VEGF agents is the most common intravitreal procedure performed by ophthalmologists. The recognition of adverse effects from the use of these medications and appropriate treatment has become increasingly important. In this paper, we will compare the inflammatory effects of the different anti-VEGF agents, differentiate their clinical features, and review the possible mechanisms involved in the development of posttreatment sterile inflammation.

\section{Definition of Sterile Endophthalmitis}

Sterile endophthalmitis (also known as "pseudoendophthalmitis") is described as any acute intraocular inflammation without infection that resolves without antibiotic treatment, unlike true endophthalmitis. A review of the literature published on PubMed between 1945 and June 2013 was conducted using combination keywords such as sterile endophthalmitis, anti-VEGF, pegaptanib, bevacizumab, ranibizumab, aflibercept, and ocular inflammation. Only the articles written in English were included. Also, in order to avoid confusion, only the studies reporting noninfectious endophthalmitis were included.

\section{Sterile Endophthalmitis versus Infectious Endophthalmitis}

Infectious endophthalmitis is the most feared complication after intravitreal injections. It is important to differentiate infectious endophthalmitis from sterile endophthalmitis, as the management and prognosis of these two entities vary vastly. While infective endophthalmitis cases are heavily treated by intravitreal antibiotics, the treatment of sterile endophthalmitis has shown prompt improvement with 
TABLE 1: Clinical characteristics of noninfectious versus infectious endophthalmitis.

\begin{tabular}{lll}
\hline & Noninfectious endophthalmitis & Infectious endophthalmitis \\
\hline Pain & $\pm[14,17,20]$ & $++[14]$ \\
Onset & $<1$ day $[10,14,16,20,21]$ to 1 week $[11,15,17,22]$ & 2.5 days (range: 1-6 days) $[14,15,23]$ \\
Signs & Blurred vision $[11]$, anterior segment inflammation & Decreased vision, severe anterior segment reaction \\
Time to resolution & greater than posterior inflammation $[10,17,18,21,22]$ & (fibrin and hypopyon), and vitritis [14] \\
Prognosis & 2-12 weeks $[11,15,17,18,20,24]$ & Extremely variable \\
\hline
\end{tabular}

TABLE 2: Sterile inflammatory rates between anti-VEGF agents.

\begin{tabular}{|c|c|c|c|c|}
\hline Study & Anti-VEGF agent & Number of patients & $\begin{array}{c}\text { Number of } \\
\text { injections }\end{array}$ & $\begin{array}{c}\text { Percentage (\%) of } \\
\text { inflammation }\end{array}$ \\
\hline Chong et al. (2010) [11] & Bevacizumab & - & 16116 & $0.40 \%$ \\
\hline Georgopoulos et al. (2009) [25] & Bevacizumab & - & 2500 & $0.03 \%$ \\
\hline Shima et al. (2008) [16] & Bevacizumab & 707 & 1300 & $0.28 \%$ \\
\hline Wickremasinghe et al. (2008) [10] & Bevacizumab & - & 1278 & $1.49 \%$ \\
\hline Johnson et al. (2010) [26] & Bevacizumab & 173 & 693 & $1.30 \%$ \\
\hline Sato et al. (2010) [22] & Bevacizumab & 35 & 35 & $14.3 \%$ \\
\hline Yamashiro et al. (2010) [19] & Bevacizumab & 15 & 20 & $73 \%$ \\
\hline Wang et al. (2013) [24] & Bevacizumab & 116 & 116 & $69 \%$ \\
\hline Wu et al. (2008) [27] & Bevacizumab & 1173 & 4303 & $0.09 \%$ \\
\hline Chong et al. (2010) [11] & Ranibizumab & - & 3839 & $0.03 \%$ \\
\hline Regillo et al. (2008) [28] & Ranibizumab & 184 & - & $0 \%$ \\
\hline Holz et al. (2011) [29] & Ranibizumab & 514 & - & $0 \%$ \\
\hline Busbee et al. (2013) [30] & Ranibizumab & 1098 & - & $0.4 \%$ \\
\hline Rosenfeld et al. (2006) [12] & Ranibizumab & 716 & - & $2.6 \%$ \\
\hline Brown et al. (2006) [13] & Ranibizumab & 280 & - & $0.35 \%$ \\
\hline Heier et al. (2006) [31] & Ranibizumab & 105 & - & $11.4 \%$ \\
\hline Antoszyk et al. (2008) [32] & Ranibizumab & 105 & - & $9.5 \%$ \\
\hline Rosenfeld et al. (2006) [18] & Ranibizumab & 29 & - & $86 \%$ \\
\hline Chun et al. (2006) [33] & Ranibizumab & 10 & 30 & $50 \%$ \\
\hline Chakravarthy et al. (2012) [34] & $\begin{array}{l}\text { Bevacizumab and } \\
\text { ranibizumab }\end{array}$ & 610 & - & $0.16 \%$ \\
\hline Ladas et al. (2009) [35] & $\begin{array}{l}\text { Bevacizumab and } \\
\text { ranibizumab }\end{array}$ & 450 & 2000 & $1.90 \%$ \\
\hline Sharma et al. (2012) [36] & $\begin{array}{l}\text { Bevacizumab and } \\
\text { ranibizumab }\end{array}$ & 524 & 1584 & $1.90 \%$ \\
\hline Hahn et al. (2013) [37] & Aflibercept & - & 30000 & $0.05 \%$ \\
\hline Ho et al. (2013) [38] & Aflibercept & 85 & - & $0 \%$ \\
\hline D’Amico et al. (2006) [39] & Pegaptanib & 1190 & - & $0 \%$ \\
\hline Singerman et al. (2008) [40] & Pegaptanib & 161 & 1254 & $9 \%$ \\
\hline
\end{tabular}

topical steroid therapy [8]. The clinical features can help when attempting to differentiate the two (Table 1).

In the literature, the incidence of sterile endophthalmitis after intravitreal anti-VEGF therapy ranges between $0.033 \%$ and $2.9 \%$ [9-14]. Meta-analyses reports have shown variability in the incidence of sterile endophthalmitis between the different anti-VEGF agents (Table 2). It typically presents 24 hours to 7 days after injection $[10,15]$, with or without pain. Pain may be an indication of the severity of the inflammation in the anterior chamber and vitreous cavity. The most common presenting symptoms are blurred vision and floaters [11].
The time between symptom presentations after injection ranges from 1 day to 1 week $[10,11,14-18]$. Visual acuity at presentation is substantially reduced compared with preinjection acuity and typically returns to preinjection acuity after resolution of the inflammation $[10,11]$. The average time to resolution of inflammation ranges from 2 to 12 weeks [11, 15, 17] and recovery of visual acuity occurs between 7 and 9 weeks [11]. Moreover, the time from injection to presentation with inflammation does not seem to affect the extent of visual recovery; it only affects the length of time to recovery [10]. In addition, history of prior intravitreal anti-VEGF injections 
does not increase the risk or severity of ocular inflammation in subsequent injections $[11,19]$.

The clinical course of sterile endophthalmitis varies based on the management of the clinical practitioner. Management includes the use of topical medications, intravitreal antibiotics, and pars plana vitrectomy with or without intravitreal antibiotics. The time to resolution based on this can vary from two to 42 days (Table 1). The median duration of inflammation was six days in patients undergoing vitrectomy, seven days in patients receiving triple intravitreal injections, and four days in patients receiving topical corticosteroids [24]. While these results may imply that treatment with topical corticosteroids is the most effective, this is not accurate. Chong et al. [11] reported only $0.27 \%, 14$ of 16166 cases, of sterile endophthalmitis resolving with topical antibiotics alone. It is difficult to generalize the treatment with the time to resolution because typically the most severe cases were chosen for pars plana vitrectomy with intravitreal antibiotics. Shah et al. [15] reported in a retrospective case series that the clinical difference between these two entities was not significant and therefore a low threshold for vitreous tap with intravitreal antibiotic injection might be warranted.

\section{Pharmacokinetics of Anti-VEGF Agents}

Before discussing possible mechanisms of inflammation after intravitreal injection, it is important to examine the pharmacokinetic properties of these agents, especially in regard to the duration of activity within the vitreous. The intravitreal halflives of bevacizumab using ELISA methods range from 4.32 to 9.82 days [41-43]. Similarly, the intravitreal half-life of ranibizumab was approximately 7.15 days [44]. In a rhesus monkey study, the vitreous half-life of pegaptanib was found to be approximately 3.9 days [45]. There are currently no reports on the pharmacokinetic properties of aflibercept using ELISA. Utilizing PET/CT to detect I-124 labeled anti-VEGF agents, Christoforidis et al. reported the intravitreal half-lives of ranibizumab, bevacizumab, and aflibercept $[46,47]$ to be 2.82 , 4.22, and 4.58 days, respectively. Their findings corroborate the previously described presence of a two-compartment pharmacokinetic decay model with an initial rapid phase followed by a slower phase described by Zou et al. [48].

\section{Antivascular Endothelial Growth Factor Drugs}

Targeting anti-VEGF in the treatment of ocular neovascular diseases first requires an understanding of the human VEGFA gene. The human VEGF-A gene is composed of eight exons with six principle amino acid isoforms $(121,145,165,183,189$, and 206) [1]. VEGF121 is freely diffusible, while VEGF189 and VEGF206 are primarily bound and sequestered in the extracellular matrix. This is due to the heparin-binding domain found in the larger isoforms of VEGF such as VEGF189 and VEGF206. VEGF165 has properties of both the diffusible and bound form of VEGF [1]. There are four anti-VEGF agents currently utilized in the treatment of ocular diseases, which differ in their isoform-binding specificities.
Pegaptanib (Macugen; Eyetech/OIS Pharmaceuticals, Melville, New York, USA) was the first anti-VEGF therapy approved for the treatment of wet AMD. It is an aptamer that selectively binds to and neutralizes VEGF-A165 while sparing smaller isoforms that lack the heparin-binding domain such as VEGF121 and VEGF110 [49]. The large-scale, randomized controlled VISION trials reported twelve cases of 1190 cases of endophthalmitis (1\%) although nine of the twelve were likely associated with violations of the injection preparation protocol, such as failure to use an eyelid speculum. In year 2 , there were four cases in 1024 patients of endophthalmitis $(0.4 \%)$ [39]. There were no reported cases of sterile endophthalmitis $[39,40]$.

Ranibizumab (Lucentis; Genentech, South San Francisco, California) was designed as a potent inhibitor of all VEGF isoforms with an affinity-matured antigen-binding fragment (Fab) derived from bevacizumab. It was developed as a Fab fragment because it was thought that its smaller-size would increase its diffusion capacity as an anti-VEGF-A agent compared to its parent bevacizumab. Ranibizumab, compared to its parent bevacizumab, has a higher affinity for VEGF with a greater potency. As an antibody-binding fragment, it lacks the domain that activates compliment-mediated cytotoxicity and Fc receptors on immune cells [50]. The primary ocular adverse event associated with ranibizumab is ocular inflammation. Many large-scale studies have reported the frequency of ocular inflammation or presumed endophthalmitis to be between 0 and $12.7 \%$. The results of these trials have been outlined below.

The rate of intraocular inflammation in the MARINA trial using ranibizumab was found to be $2.6 \%$ [12]. In the PIER study, no cases of serious uveitis or endophthalmitis were noted with the use of ranibizumab [28]. In the IVAN trial, only one case of $610(0.1 \%)$ developed uveitis [34]. The HARBOR study group reported a $0.7 \%$ rate of endophthalmitis and a $0.4 \%$ rate of inflammation with intravitreal ranibizumab [30]. The SUSTAIN study utilized ranibizumab and reported no episodes of sterile endophthalmitis in the 249 patients studied [29]. The first year results of the FOCUS study revealed that the more frequently associated serious ocular adverse events were intraocular inflammation (11.4\%) and endophthalmitis (1.9\%; 4.8\% including presumed cases) [31]. In the two-year FOCUS study, endophthalmitis and serious ocular inflammation occurred in $2.9 \%$ and $12.4 \%$, respectively, in the ranibizumab + PDT patient groups [32]. It should be noted that this study used a lyophilized formulation of ranibizumab that was discontinued afterwards. Among the 280 patients treated in the ANCHOR trial, presumed endophthalmitis occurred in 2 patients $(0.7 \%)$ and serious uveitis occurred in 1 patient $(0.35 \%)$ at year 1 [13]. Ladas et al. reported a $1.9 \%$ frequency of ocular inflammation after intravitreal ranibizumab and bevacizumab injection with no reported statistical difference between the two medications [35]. Overall, the rates of presumed endophthalmitis or severe inflammation were similar between the two drugs.

Bevacizumab (Avastin; Roche, Basel, Switzerland) was initially a drug approved by the Food and Drug Administration for the treatment of glioblastoma, metastatic colon cancer, advanced nonsquamous non-small cell lung 
cancer, and metastatic kidney disease. It is a full-length murine-derived humanized, monoclonal, nonselective antibody against VEGF-A. It is a significantly larger molecule with potentially less effective retinal penetration and binding affinity to VEGF. In comparison to ranibizumab (Lucentis), which is an antibody fragment, bevacizumab has an Fc fragment which may make it more immunogenic or proinflammatory. Larger molecules with Fc constant fragments and antibody-binding Fab fragments are more immunogenic than those with the antibody-binding fragment alone.

A retrospective single center study conducted by Johnson et al. reported the incidence of intraocular inflammation after bevacizumab injection to be $1.3 \%$ after 693 injections [26]. Similarly, Georgopoulos et al. reported a $0.3 \%$ incidence of intraocular inflammation after 2500 injections of bevacizumab [25] and Shima et al. reported a $0.2 \%$ incidence of ocular inflammation after 1300 injections [16]. In a smaller study that used the same lot of bevacizumab, 5 of the 35 (14.3\%) of the patients developed severe intraocular inflammation [22]. In this study however, $80 \%$ of the patients had received bevacizumab injections previously without an intraocular inflammatory episode. A similar incidence of lot specific intraocular inflammation has been reported in China where 80 patients of $116(69 \%)$ developed postinjection intraocular inflammation [24]. This study implicated endotoxin as the cause of the inflammation.

Aflibercept (Eyelea, Regeneron, Inc., Tarrytown, NY) utilizes the fusion of multiple endogenous receptor components creating what is called a VEGF Trap. It binds with higher affinity to multiple isoforms of VEGF-A as well as VEGFR1 ligands, VEGF-B, and placental growth factor (PIGF). Consistent with this higher affinity, VEGF Trap demonstrates a higher ability in blocking VEGF-mediated mobilization and migration of human endothelial cells [51].

It was approved by the FDA in November 2011, and within the first three months after its approval, a cluster of small cases were reported with injection-related ocular inflammation [37]. This report indicated that aflibercept was associated with sterile inflammation in $0.05 \%$ of cases and associated with pain far more than the other anti-VEGF agents (60\%). Prior to approval of aflibercept, the clinical characteristic of pain could often be used to distinguish between sterile inflammation and endophthalmitis. This report has led clinicians to be more cautious and more apt to treat with intravitreal antibiotics sooner. Subgroup analysis in this study did not detect any variables significantly affecting visual outcome or number of days to resolution. Moreover, Ho et al. looked at the short-term outcomes of aflibercept in 245 patients for five months and reported no cases of endophthalmitis [38]. More recently, The American Society of Retina Specialists Therapeutic Surveillance Committee (ASRS TSC) reported at the annual ASRS meeting in August 2013 that there were at least 41 cases of sterile endophthalmitis among more than 800,000 aflibercept injections given in the United States between December 2011 and June 2013. While some of these reported cases responded to topical corticosteroid treatment and observation alone, others were associated with more severe inflammation that resembled infectious endophthalmitis. The ASRS TSC concluded that there was no clear pattern detected to predict these events.

\section{Hypotheses}

There are several hypotheses pertaining to the etiology of sterile inflammation secondary to intravitreal anti-VEGF injections. The manufacturer's guidelines for anti-VEGF agent preparation state that the medication should be refrigerated at 2 to 8 degrees $\mathrm{C}$ (36 to 46 degrees F), protected from the light, stored in the original carton until used, and used within 8 hours of being opened [10]. Any variance from this protocol could result in degradation of the agent with increased immunogenicity $[52,53]$.

The eye may mount an immune response to the antibody molecule after prior exposure to the drug. One report found an $83 \%$ incidence of sterile inflammation after intravitreal injection of ranibizumab. One of the 29 patients $(0.03 \%)$ had to be permanently withdrawn from the study secondary to the severe inflammation. In this study, the inflammation was low-grade and self-limited and did not increase with repeated injections or increasing doses [18]. While these results do not support this immune mediated hypothesis, the immunogenicity varies between different anti-VEGF agents.

Bacterial endotoxin contamination has been reported in the pharmaceutical production phase of antibody preparation $[24,54]$. In a study by Wang et al., a total of 116 patients were injected from 3 vials of counterfeit bevacizumab with 80 patients subsequently developing intraocular inflammation. The presence of endotoxin in vitreous specimens was confirmed by laboratory testing. They concluded that endotoxin testing should be considered as part of the laboratory investigation in patients who develop noninfectious inflammation. These studies demonstrated that endotoxin contamination of individual aliquots is possible during preparation. While this occurrence could explain clusters of sterile endophthalmitis cases in patients treated with injections from the same batch, it is unlikely to explain the cause of sporadic cases.

While systemic use of anti-VEGF agents has not been described to entice an inflammatory response, in the closed system of the eye, it may mount a significant response to the anti-VEGF. Multiple case series have described a high percentage (35-78\%) of sterile ocular inflammation after intravitreal injections from a single lot of anti-VEGF agents.

Yamashiro et al. reported 14 consecutive cases of endophthalmitis after intravitreal injection of bevacizumab from the same lot. Of the 19 eyes, 14 showed ocular inflammation after injection. Vitreous samples from these patients revealed the etiology to be noninfectious [19]. Similarly, in a report by Sato et al., 14.3\% (five of 35 cases) were noted to develop a severe intraocular inflammation after intravitreal injection of bevacizumab. Vitrectomy was performed in all 5 cases with no growth of any causative organisms or microbes [22]. All five cases were resolved with treatment with steroids. These reports support the possibility of trace endotoxin contamination resulting in sterile endophthalmitis. Wang et al. published a retrospective paper where 116 patients were injected with counterfeit bevacizumab. Of these patients, $69 \%$ developed a sterile endophthalmitis with endotoxin levels (endotoxin units) detected as high as $36 \mathrm{Eu} / \mathrm{mL}$ (standard of bevacizumab $<2 \mathrm{Eu} / \mathrm{mL}$ ) [24]. These patients shared the typical clinical features described above with $78 \%$ of the affected patients returning to pre-injection visual acuity. 
In summary, sterile inflammation is an adverse event of intravitreal anti-VEGF injection that should be included in the patient consent in all anti-VEGF agents. Acute intraocular inflammation is most frequently following bevacizumab [36], possibly due to the less stringent purification process of the medication. In most cases, the inflammation resolves and vision returns to baseline. A history of prior inflammation does not increase the risk with subsequent injections.

\section{Conflict of Interests}

The authors declare that there is no competing/conflict of interests related to any topic in this paper.

\section{References}

[1] N. Ferrara, "Vascular endothelial growth factor: basic science and clinical progress," Endocrine Reviews, vol. 25, no. 4, pp. 581611, 2004.

[2] P. J. Rosenfeld, A. A. Moshfeghi, and C. A. Puliafito, "Optical coherence tomography findings after an intravitreal injection of bevacizumab (Avastin) for neovascular age-related macular degeneration," Ophthalmic Surgery Lasers and Imaging, vol. 36, no. 4, pp. 331-335, 2005.

[3] P. J. Rosenfeld, A. E. Fung, and C. A. Puliafito, "Optical coherence tomography findings after an intravitreal injection of bevacizumab (Avastin) for macular edema from central retinal vein occlusion," Ophthalmic Surgery Lasers and Imaging, vol. 36, no. 4, pp. 336-339, 2005.

[4] B. P. Nicholson and A. P. Schachat, "A review of clinical trials of anti-VEGF agents for diabetic retinopathy," Graefe's Archive for Clinical and Experimental Ophthalmology, vol. 248, no. 7, pp. 915-930, 2010.

[5] K. G. Falavarjani, M. Modarres, and H. Nazari, "Therapeutic effect of bevacizumab injected into the silicone oil in eyes with neovascular glaucoma after vitrectomy for advanced diabetic retinopathy," Eye, vol. 24, no. 4, pp. 717-719, 2010.

[6] H. Nazari, M. Modarres, M. M. Parvaresh, and K. Ghasemi Falavarjani, "Intravitreal bevacizumab in combination with laser therapy for the treatment of severe retinopathy of prematurity (ROP) associated with vitreous or retinal hemorrhage," Graefe's Archive for Clinical and Experimental Ophthalmology, vol. 248, no. 12, pp. 1713-1718, 2010.

[7] E. T. Detorakis, G. Agorogiannis, E. E. Drakonaki, M. K. Tsilimbaris, and I. G. Pallikaris, "Successful management of choroidal metastasis with intravitreal ranibizumab injection," Ophthalmic Surgery Lasers Imaging, vol. 43, pp. e47-e51, 2012.

[8] C. N. Kay, R. M. Tarantola, K. M. Gehrs et al., "Uveitis following intravitreal bevacizumab: A non-infectious cluster," Ophthalmic Surgery Lasers and Imaging, vol. 42, no. 4, pp. 292-296, 2011.

[9] L. H. Lima, S. A. Zweifel, M. Engelbert et al., "Evaluation of safety for bilateral same-day intravitreal injections of antivascular endothelial growth factor therapy," Retina, vol. 29, no. 9, pp. 1213-1217, 2009.

[10] S. S. Wickremasinghe, K. Michalova, J. Gilhotra et al., "Acute Intraocular Inflammation after Intravitreous Injections of Bevacizumab for Treatment of Neovascular Age-related Macular Degeneration," Ophthalmology, vol. 115, no. 11, pp. 1911.el1915.e1, 2008.

[11] D. Y. Chong, R. Anand, P. D. Williams, J. A. Qureshi, and D. G. Callanan, "Characterization of sterile intraocular inflammatory responses after intravitreal bevacizumab injection," Retina, vol. 30, no. 9, pp. 1432-1440, 2010.

[12] P. J. Rosenfeld, D. M. Brown, J. S. Heier et al., "Ranibizumab for neovascular age-related macular degeneration," New England Journal of Medicine, vol. 355, no. 14, pp. 1419-1431, 2006.

[13] D. M. Brown, P. K. Kaiser, M. Michels et al., "Ranibizumab versus verteporfin for neovascular age-related macular degeneration," New England Journal of Medicine, vol. 355, no. 14, pp. 14321444, 2006.

[14] M. Tolentino, "Systemic and Ocular Safety of Intravitreal AntiVEGF Therapies for Ocular Neovascular Disease," Survey of Ophthalmology, vol. 56, no. 2, pp. 95-113, 2011.

[15] C. P. Shah, S. J. Garg, J. F. Vander, G. C. Brown, R. S. Kaiser, and J. A. Haller, "Outcomes and risk factors associated with endophthalmitis after intravitreal injection of anti-vascular endothelial growth factor agents," Ophthalmology, vol. 118, no. 10, pp. 20282034, 2011.

[16] C. Shima, H. Sakaguchi, F. Gomi et al., "Complications in patients after intravitreal injection of bevacizumab," Acta Ophthalmologica, vol. 86, no. 4, pp. 372-376, 2008.

[17] S. J. Bakri, T. A. Larson, and A. O. Edwards, "Intraocular inflammation following intravitreal injection of bevacizumab," Graefe's Archive for Clinical and Experimental Ophthalmology, vol. 246, no. 5, pp. 779-781, 2008.

[18] P. J. Rosenfeld, J. S. Heier, G. Hantsbarger, and N. Shams, “Tolerability and Efficacy of Multiple Escalating Doses of Ranibizumab (Lucentis) for Neovascular Age-Related Macular Degeneration," Ophthalmology, vol. 113, no. 4, pp. 623.e1-632.e1, 2006.

[19] K. Yamashiro, A. Tsujikawa, K. Miyamoto et al., "Sterile endophthalmitis after intravitreal injection of bevacizumab obtained from a single batch," Retina, vol. 30, no. 3, pp. 485490, 2010.

[20] A. Mozayan and S. Farah, "Acute anterior uveitis following intravitreal injection of bevacizumab," Ophthalmic Surgery Lasers Imaging Retina, vol. 44, no. 1, pp. 25-27, 2013.

[21] C. N. Kay, R. M. Tarantola, K. M. Gehrs et al., "Uveitis following intravitreal bevacizumab: a non-infectious cluster," Ophthalmic Surgery Lasers and Imaging, vol. 42, no. 4, pp. 292-296, 2011.

[22] T. Sato, K. Emi, T. Ikeda et al., "Severe Intraocular Inflammation after Intravitreal Injection of Bevacizumab," Ophthalmology, vol. 117, no. 3, pp. 512.e2-516.e2, 2010.

[23] D. Johnson and S. Sharma, "Ocular and systemic safety of bevacizumab and ranibizumab in patients with neovascular agerelated macular degeneration," Current Opinion in Ophthalmology, vol. 24, no. 3, pp. 205-212, 2013.

[24] F. Wang, S. Yu, K. Liu et al., "Acute intraocular inflammation caused by endotoxin after intravitreal injection of counterfeit bevacizumab in Shanghai, China," Ophthalmology, vol. 120, pp. 355-361, 2013.

[25] M. Georgopoulos, K. Polak, F. Prager, C. Prünte, and U. Schmidt-Erfurth, "Characteristics of severe intraocular inflammation following intravitreal injection of bevacizumab (Avastin)," British Journal of Ophthalmology, vol. 93, no. 4, pp. 457462, 2009.

[26] D. Johnson, H. Hollands, S. Hollands, and S. Sharma, "Incidence and characteristics of acute intraocular inflammation after intravitreal injection of bevacizumab: A retrospective cohort study," Canadian Journal of Ophthalmology, vol. 45, no. 3, pp. 239-242, 2010.

[27] L. Wu, M. A. Martínez-Castellanos, H. Quiroz-Mercado et al., "Twelve-month safety of intravitreal injections of bevacizumab 
(Avastin): Results of the pan-american collaborative retina study group (PACORES)," Graefe's Archive for Clinical and Experimental Ophthalmology, vol. 246, no. 1, pp. 81-87, 2008.

[28] C. D. Regillo, D. M. Brown, P. Abraham et al., "Randomized, double-masked, sham-controlled trial of ranibizumab for neovascular age-related macular degeneration: PIER Study year 1," American Journal of Ophthalmology, vol. 145, no. 2, pp. 239-e5, 2008.

[29] F. G. Holz, W. Amoaku, J. Donate et al., "Safety and efficacy of a flexible dosing regimen of ranibizumab in neovascular agerelated macular degeneration: The SUSTAIN study," Ophthalmology, vol. 118, no. 4, pp. 663-671, 2011.

[30] B. G. Busbee, A. C. Ho, D. M. Brown et al., "Twelve-Month Efficacy and Safety of $0.5 \mathrm{mg}$ or $2.0 \mathrm{mg}$ Ranibizumab in Patients with Subfoveal Neovascular Age-related Macular Degeneration for the HARBOR study group," Ophthalmology, vol. 120, pp. 1046-1056, 2013.

[31] J. S. Heier, D. S. Boyer, T. A. Ciulla et al., "Ranibizumab combined with verteporfin photodynamic therapy in neovascular age-related macular degeneration: Year 1 results of the FOCUS study," Archives of Ophthalmology, vol. 124, no. 11, pp. 1532-1542, 2006.

[32] A. N. Antoszyk, L. Tuomi, C. Y. Chung, and A. Singh, "Ranibizumab combined with verteporfin photodynamic therapy in neovascular age-related macular degeneration (FOCUS): year 2 results," American Journal of Ophthalmology, vol. 145, no. 5, pp. 862.e3-874.e3, 2008.

[33] D. W. Chun, J. S. Heier, T. M. Topping, J. S. Duker, and J. M. Bankert, "A Pilot Study of Multiple Intravitreal Injections of Ranibizumab in Patients with Center-Involving Clinically Significant Diabetic Macular Edema," Ophthalmology, vol. 113, no. 10, pp. 1706-1712, 2006.

[34] U. Chakravarthy, S. P. Harding, C. A. Rogers et al., "Ranibizumab versus bevacizumab to treat neovascular age-related macular degeneration: one-year findings from the IVAN randomized trial," Ophthalmology, vol. 119, pp. 1399-1411, 2012.

[35] I. D. Ladas, D. A. Karagiannis, A. A. Rouvas, A. I. Kotsolis, A. Liotsou, and I. Vergados, "Safety of repeat intravitreal injections of bevacizumab versus ranibizumab: Our experience after 2,000 injections," Retina, vol. 29, no. 3, pp. 313-318, 2009.

[36] S. Sharma, D. Johnson, M. Abouammoh, S. Hollands, and A. Brissette, "Rate of serious adverse effects in a series of bevacizumab and ranibizumab injections," Canadian Journal of Ophthalmology, vol. 47, no. 3, pp. 275-279, 2012.

[37] P. Hahn, J. E. Kim, and S. Stinnett, "Aflibercept-Related Sterile Inflammation," Ophthalmology, vol. 120, no. 5, pp. 1100.5-1101.5, 2013.

[38] V. Y. Ho, S. Yeh, T. W. Olsen et al., "Short-Term Outcomes of Aflibercept for Neovascular Age-Related Macular Degeneration in Eyes Previously Treated With Other Vascular Endothelial Growth Factor Inhibitors," American Journal of Ophthalmology, vol. 156, pp. 23-28, 2013.

[39] D. J. D’Amico, H. N. Masonson, M. Patel et al., "Pegaptanib sodium for neovascular age-related macular degeneration. Two-year safety results of the two prospective, multicenter, controlled clinical trials," Ophthalmology, vol. 113, no. 6, pp. 992.e11001.e1, 2006

[40] L. J. Singerman, H. Masonson, M. Patel et al., "Pegaptanib sodium for neovascular age-related macular degeneration: third-year safety results of the VEGF inhibition study in ocular neovascularisation (VISION) trial," British Journal of Ophthalmology, vol. 92, no. 12, pp. 1606-1611, 2008.
[41] S. J. Bakri, M. R. Snyder, J. M. Reid, J. S. Pulido, and R. J. Singh, "Pharmacokinetics of Intravitreal Bevacizumab (Avastin)," Ophthalmology, vol. 114, no. 5, pp. 855-859, 2007.

[42] Q. Zhu, F. Ziemssen, S. Henke-Fahle et al., "Vitreous Levels of Bevacizumab and Vascular Endothelial Growth Factor-A in Patients with Choroidal Neovascularization," Ophthalmology, vol. 115, no. 10, pp. 1750-e1, 2008.

[43] T. U. Krohne, N. Eter, F. G. Holz, and C. H. Meyer, "Intraocular Pharmacokinetics of Bevacizumab After a Single Intravitreal Injection in Humans," American Journal of Ophthalmology, vol. 146, no. 4, pp. 508-512, 2008.

[44] T. U. Krohne, Z. Liu, F. G. Holz, and C. H. Meyer, "Intraocular pharmacokinetics of ranibizumab following a single intravitreal injection in humans," American Journal of Ophthalmology, vol. 154, no. 4, pp. 682-686, 2012.

[45] D. W. Drolet, J. Nelson, C. E. Tucker et al., "Pharmacokinetics and safety of an anti-vascular endothelial growth factor aptamer (NX1838) following injection into the vitreous humor of rhesus monkeys," Pharmaceutical Research, vol. 17, no. 12, pp. 1503$1510,2000$.

[46] J. B. Christoforidis, M. M. Carlton, M. V. Knopp, and G. H. Hinkle, "PET/CT imaging of I-124-radiolabeled bevacizumab and ranibizumab after intravitreal injection in a rabbit model," Investigative Ophthalmology and Visual Science, vol. 52, no. 8, pp. 5899-5903, 2011.

[47] J. B. Christoforidis, M. M. Williams, S. Kothandaraman, K. Kumar, F. J. Epitropoulos, and M. V. Knopp, "Pharmacokinetic Properties of Intravitreal I-124-Aflibercept in a Rabbit Model Using PET/CT," Current Eye Research, vol. 37, no. 12, pp. 11711174, 2012.

[48] P. Zou, S. P. Povoski, N. C. Hall et al., "124I-HuCC49deltaCH2 for TAG-72 antigen-directed positron emission tomography (PET) imaging of LS174T colon adenocarcinoma tumor implants in xenograft mice: Preliminary results," World Journal of Surgical Oncology, vol. 8, article 65, 2010.

[49] E. W. M. Ng, D. T. Shima, P. Calias, E. T. Cunningham Jr., D. R. Guyer, and A. P. Adamis, "Pegaptanib, a targeted anti-VEGF aptamer for ocular vascular disease," Nature Reviews Drug Discovery, vol. 5, no. 2, pp. 123-132, 2006.

[50] N. Ferrara, L. Damico, N. Shams, H. Lowman, and R. Kim, "Development of ranibizumab, an anti-vascular endothelial growth factor antigen binding fragment, as therapy for neovascular age-related macular degeneration," Retina, vol. 26, no. 8, pp. 859-870, 2006.

[51] N. Papadopoulos, J. Martin, Q. Ruan et al., "Binding and neutralization of vascular endothelial growth factor (VEGF) and related ligands by VEGF Trap, ranibizumab and bevacizumab," Angiogenesis, vol. 15, no. 2, pp. 171-185, 2012.

[52] E. Hochuli, "Interferon immunogenicity: technical evaluation of interferon- $\alpha 2 \mathrm{a}$," Journal of Interferon and Cytokine Research, vol. 17, no. 1, pp. S15-S21, 1997.

[53] E. Koren, L. A. Zuckerman, and A. R. Mire-Sluis, "Immune responses to therapeutic proteins in humans-Clinical significance, assessment and prediction," Current Pharmaceutical Biotechnology, vol. 3, no. 4, pp. 349-360, 2002.

[54] D. G. Remick, R. G. Kunkel, J. W. Larrick, and S. L. Kunkel, "Acute in vivo effects of human recombinant tumor necrosis factor," Laboratory Investigation, vol. 56, no. 6, pp. 583-590, 1987. 


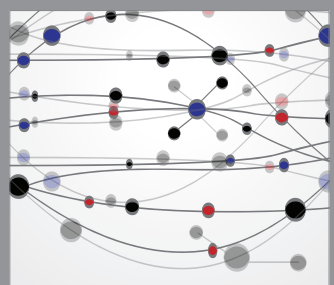

The Scientific World Journal
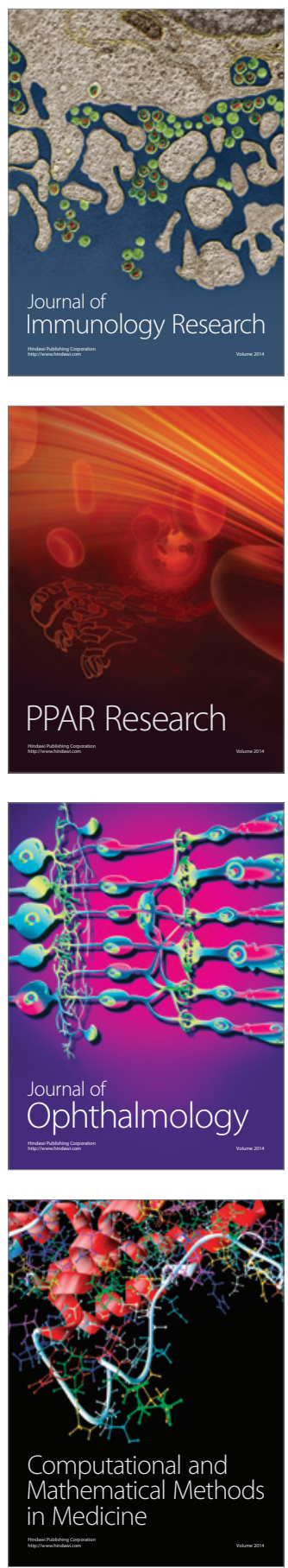

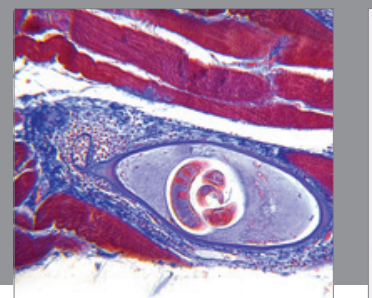

Gastroenterology

Research and Practice
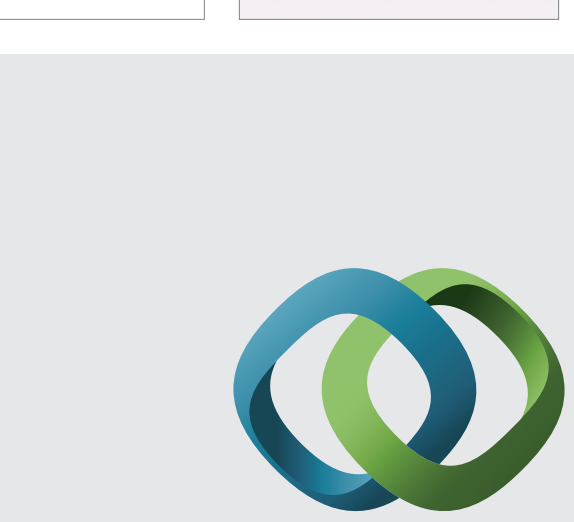

\section{Hindawi}

Submit your manuscripts at

http://www.hindawi.com
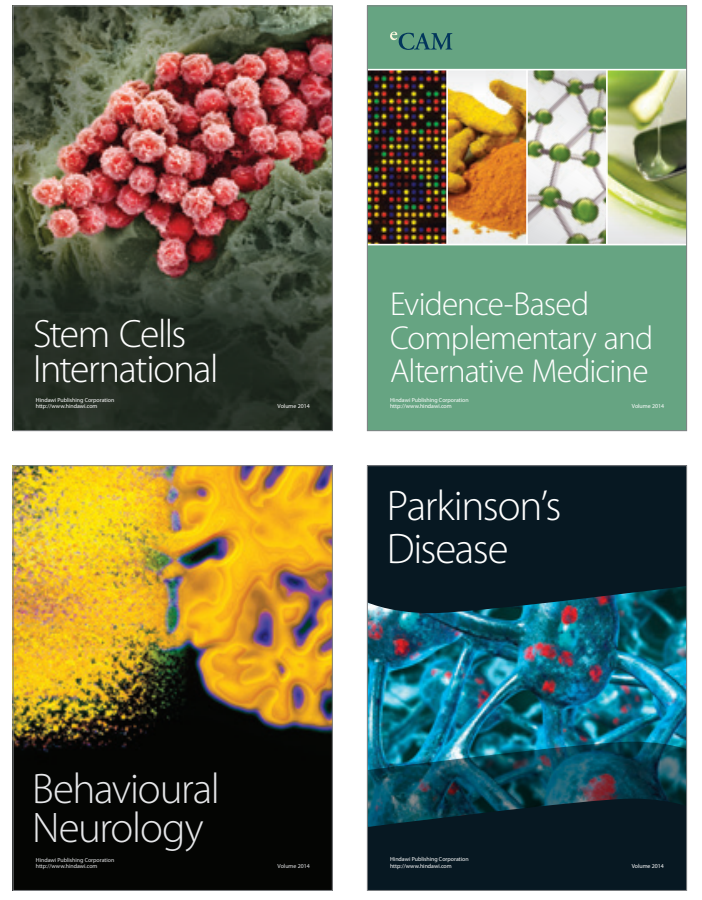
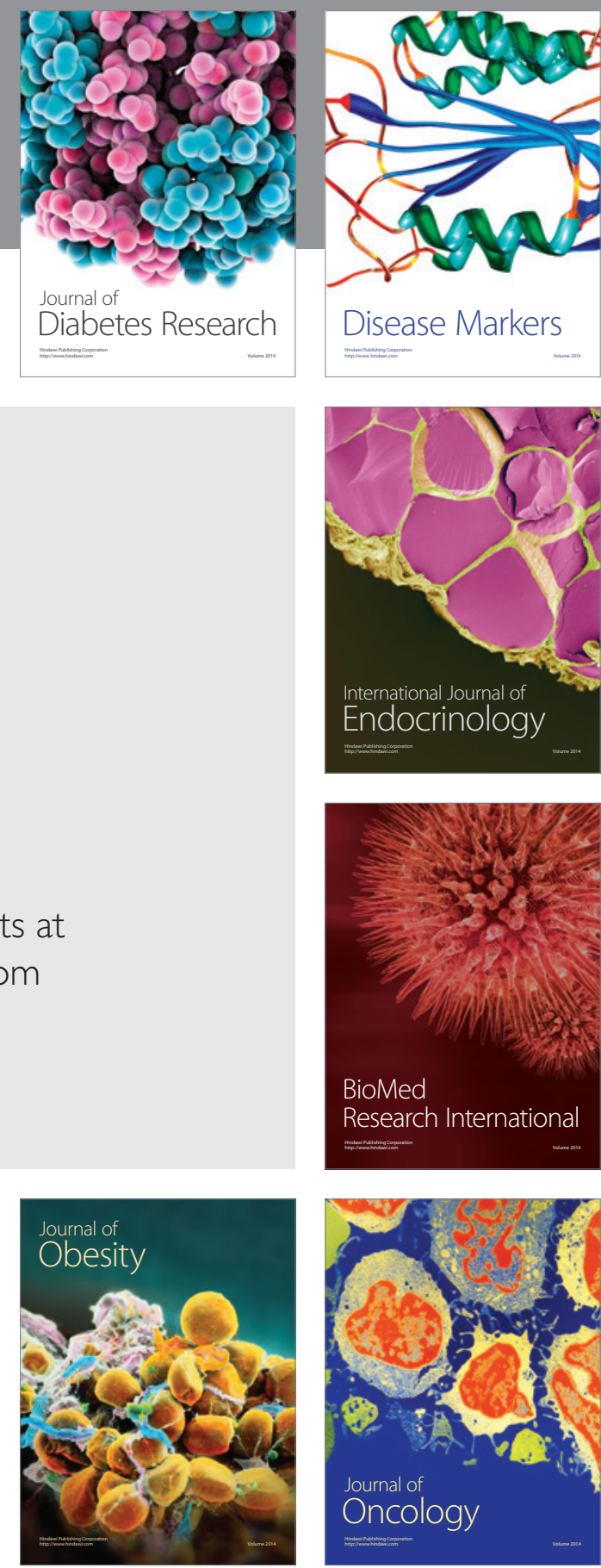

Disease Markers
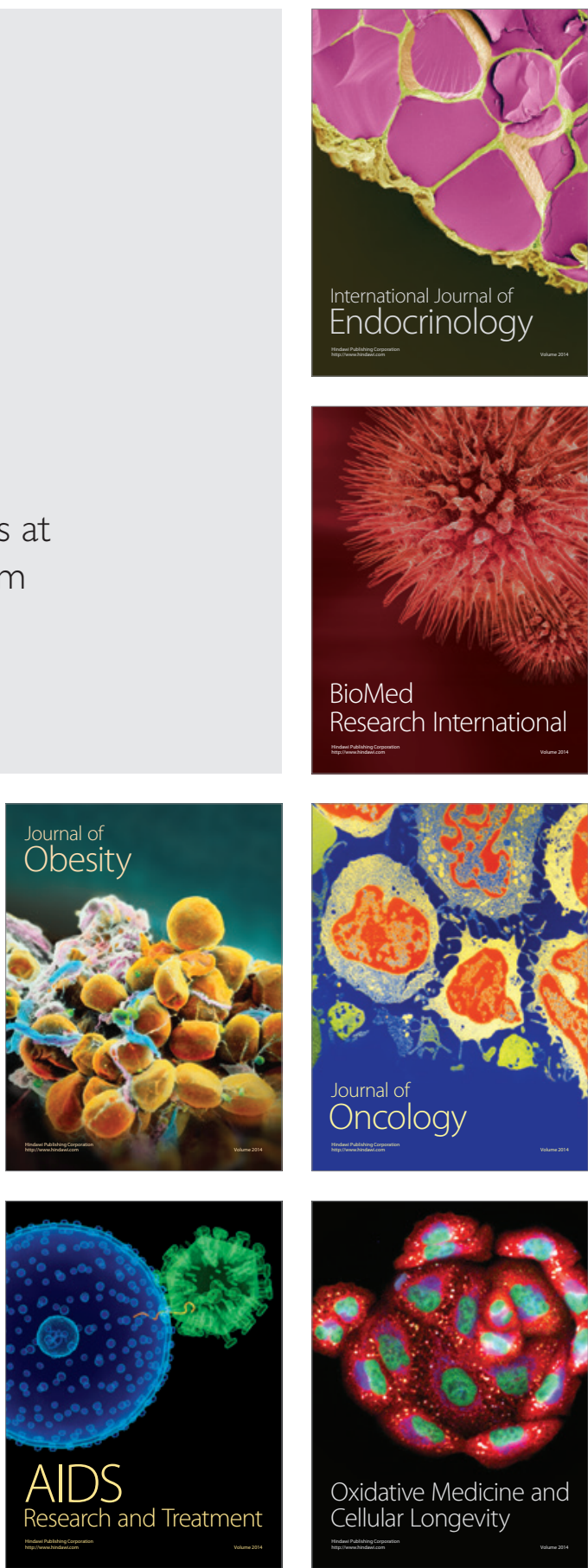